\title{
High Accuracy Phishing Detection Based on Convolutional Neural Network
}

${ }^{1}$ AjithKumar Reddy K, ${ }^{2}$ Darshith M P, ${ }^{3}$ Divya Megha H S, ${ }^{4}$ Omshree V, ${ }^{5}$ Sudhakara Reddy M

1,2,3,4 Student, Department of Computer Science and Engineering, Nagarjuna College of

Engineering and Technology, Bengaluru, Karnataka, India.

${ }^{5}$ Assistant Professor, Department of Computer Science and Engineering, Nagarjuna College

of Engineering and Technology, Bengaluru, Karnataka, India

Article Info

Volume 8, Issue 3

Page Number : $454-457$

\section{Publication Issue}

May-June-2021

\section{Article History}

Accepted : 25 May 2021

Published : 31 May 2021

\section{ABSTRACT}

There are numerous web security dangers yet one of the significant web security issues is Phishing sites that focus on the human weaknesses instead of programming weaknesses. It tends to be depicted as the way toward acquiring the online clients to get their touchy data, for example, usernames and passwords. These days, phishing is one of the greatest regular web dangers as for the critical increase of the World Wide Web in volume over the long run. Phishing aggressors consistently utilize new (multi day) and complex procedures to beguile online clients. Thus, it is important that the counter phishing framework is ongoing and quick and furthermore influences from a shrewd phishing recognition arrangement. Here, we build up a very much established location framework which can adaptively coordinate with the changing climate and phishing sites. Our strategy is an on the web and highlight rich AI procedure to separate the phishing and real sites. Since the proposed approach removes various sorts of various highlights from URLs and pages source code, it is a totally customer side arrangement and doesn't need any assistance from the outsider. In this task, we offer a clever framework for finding phishing sites. The framework depends on an AI technique, explicitly managed learning. We have chosen the Logistic Regression strategy because of its great presentation in grouping. Our point is to acquire a better classifier by considering the attributes of phishing site and pick the better mix of them to prepare the grouped.

Keywords : Phishing website, anti-phishing, Logistic regression technique.

\section{INTRODUCTION}

Each exploration identified with phishing assaults has been enormously centered on arrangement leaving the wrongdoing and issue unknown. The writing additionally distinguished that giving the arrangement without investigating the issue isn't the best approach to deal with this danger. Consequently,

Copyright: () the author(s), publisher and licensee Technoscience Academy. This is an open-access article distributed under the terms of the Creative Commons Attribution Non-Commercial License, which permits unrestricted non-commercial use, distribution, and reproduction in any medium, provided the original work is properly cited 
a CRI approach is proposed to investigate the wrongdoing factor, audit on counteraction methods and examine the holes. It is fundamental for carry out a CRI way to deal with assistance the future exploration by adding another wellspring of writing. The point is to help the new analyst and fortify the phishing wrongdoing writing survey, the CRI approach will ultimately bring about an allencompassing enemy of phishing writing audit system. Wrongdoing has expanded inside the blast of Information innovation, internet providers and advanced hardware as crooks have utilized those devices and conditions on the internet just as in reality. Ordinary digital wrongdoing is web wrongdoing, for example, charge card extortion, parody site, infection spreading and organization interruption and hacking. This paper accentuates on perhaps the most concerned digital wrongdoing for example Phishing. As of now, millions of Internet clients convey either close to home or business levels are giving a chance to phisher to hoodwink them without any problem.

This paper consists of selecting optimal algorithm for finding fraud pattern through effective comparison of machine learning techniques through an active performance measure for detection of fraudulent phishing. The rest of this paper is presented as follows. Section II presents the related works. Section III presents the methodology. Finally, the conclusion and discussion in Section IV.

\section{LITERATURE SERVEY}

The Web has become a stage for supporting a wide scope of criminal undertakings like spam promoted business, monetary misrepresentation and as a vector for spreading malware. The quick development of Word Wide Web and the Internet-based advances change the inclination of clients from customary shopping to electronic trade. These days, numerous hoodlums center around the internet to discover their casualties for certain particular stunts for example, phishing. The phishing assault is a fake endeavor or data fraud where the assailant bamboozles casualties to utilize a malevolent site, the look and feel of which is indistinguishable from the real one. The phishers endeavor to get their casualties' pivotal data like passwords, account subtleties, Visa numbers, usernames, passwords, and so forth At the end of the day, phishing is an illustration of social designing strategies being utilized to bamboozle clients. Clients are frequently baited by interchanges indicating to be from confided in gatherings like social sites, sell off destinations, banks, online installment processors or IT directors. This kind of web assault begins with a deceitful email or other correspondence as a weapon that is intended to bait a casualty. The message is made to look like it comes from a confided in sender, and furthermore the presence of their noxious sites are like the confided in site.

\section{SYSTEMDESIGN}

Frameworks configuration is the way toward characterizing the design, parts, modules, interfaces, and information for a framework to fulfill determined necessities. Frameworks configuration could consider it to be the utilization of frameworks hypothesis to item advancement. There is some cover with the orders of frameworks examination, frameworks design and frameworks designing. In the event that the more extensive subject of item advancement "mixes the point of view of promoting, plan, and fabricating into a solitary way to deal with item advancement," at that point configuration is the demonstration of taking the showcasing data and making the plan of the item to be fabricated. Frameworks configuration is hence the way toward characterizing and creating frameworks to fulfill determined necessities of the client. Until the 1990s frameworks configuration had a urgent and regarded job in the information handling industry. During the 1990s normalization of equipment and programming 
brought about the capacity to fabricate measured frameworks. The expanding significance of programming running on nonexclusive stages has upgraded the control of computer programming. Article situated examination and plan technique a returning into the most generally utilized strategies for PC frameworks design.[citation needed]. The UM has become the standard language in object oriented investigation and design.[citation needed] It is generally utilized for displaying programming frameworks and is progressively utilized for high planning non- programming frameworks and organizations.[citation needed] Framework configuration is quite possibly the main periods of programming advancement measure. The reason for the plan is to design the arrangement of an issue determined by the prerequisite documentation. In other words the initial phase in the answer for the issue is the plan of the undertaking. The plan of the framework is maybe the most basic factor influencing the nature of the product. The goal of the plan stage is to create by and large plan of the product. It means to sort out the modules that ought to be in the framework to satisfy all the framework necessities in a proficient way. The plan will contain the detail of every one of these modules, their collaboration with different modules and the ideal yield from every module. The yield of the plan cycle is a depiction of the product design.

\section{PROPOSED SYSTEM}

We propose to build up an application which can anticipate the weakness of a phishing sites given fundamental information like URL length, area length and so forth. We propose to gather applicable information relating all components identified with our field of study, train the information according to the proposed calculation of AI and foresee how solid there is a chance for a site to be a phishing site. To execute the AI model utilizing Python to foresee the phishing site from a given URL. Train the model against sufficient informational collections to keep up the precision level above 90\%. Advantages of the proposed system are

- $\quad$ Proven high accuracy
- $\quad$ Solution made available to public over the
cloud in as-a-service model

\section{PHISHING LIFE CYCLE}

Stage 1: Plan and Setup

This is the main period of the phishing lifecycle, whereby the aggressors distinguish the objective association, an individual or a country to be assaulted. They uncover the fundamental insights about their objective and its organization, basically by actually visiting the spot/individual, or by observing the traffic going all through the objective organization. They at that point set up the assaults by sending practical methods like site, messages containing malignant connections, and so on These instruments essentially divert the casualty to the false website page

\section{Stage 2: Phishing}

This is the second period of the phishing lifecycle where the real movement happens. The assailants send mock messages to the casualty utilizing gathered email tends to which request private data from the person in question. They by and large camouflage as some trustworthy financial association that needs the casualty's very own data to update their records and the casualty should react critically by tapping on some malevolent connection.

\section{Stage 3: Break-in/Infiltration}

In this stage, the casualty taps on the noxious connection and when he does that, a malware naturally introduces on his gadget that permits the aggressor to get to the framework and barge in, change its setups and access rights to it. At times tapping on the noxious connection may likewise 
prompt some phony page that requests secret data from the person in question.

\section{Stage 4: Data Collection}

When the assailants access the casualty's framework, they separate the necessary information. On the off chance that the casualty gives classified record subtleties, the assailant would then be able to get to his record, which may ultimately prompt monetary misfortunes to the person in question. On account of malware assaults, the aggressors acquire distant admittance to the casualty's framework and concentrate the necessary information or roll out any improvements according to will.

\section{Stage 5: Break-out/Exfiltration}

This last period of the phishing lifecycle includes exfiltration. When the aggressors approach and acquired the necessary data, they eliminate all the proof, for example, the bogus site accounts. They at that point track the level of accomplishment of their assault to refine their future assaults.

\section{MODEL IMPLEMENTATION}

This module carries out the accompanying Logistic Regression AI calculation to distinguish if the inputted URL is a phishing site or not calculated relapse is a measurable model that in its essential structure utilizes a strategic capacity to display a parallel ward variable, albeit a lot more perplexing expansions exist. In relapse examination, calculated relapse (or logit relapse) is assessing the boundaries of a strategic model (a type of parallel relapse).

\section{VII.CONCLUSION AND FUTURE SCOPE}

Phishing is turning into an always developing danger to clients as the assaults advance and become harder to recognize. The lawbreakers who complete these assaults are progressively difficult to get. To battle these difficulties, we have proposed a three-pronged methodology. The utilization of a filtration framework diminishes the quantity of phishing messages that arrive at the client, diminishing the odds that they will be phished. The UI model gives clients admonitions when the site they are visiting isn't trusted, thusly shielding against the opportunity that persuading email has driven them to a phishing site. At last, by drawing in clients with educative games implanted preparing, the actual clients can begin to rehearse strategies for forestalling phishing. Despite the fact that assailants continue refreshing phishing strategies and it's turning into a more mind boggling assignment to forestall and distinguish phishing, keeping awake to date with AI based computerized guards in these three classifications in our proposed arrangement approach will actually want to help monitor phishing.

\section{REFERENCES}

[1]. Phishlabs, "2019 Phishing Trends and Intelligence Report: The Growing Social Engineering Threat" 2019, [online] availableat: https://www.phishlabs.com.

[2]. K. Jain and B. B. Gupta, "An epic way to deal with ensure against phishing assaults at customer side utilizing autorefreshed white-list," EURASIP Journal on Information Security, vol. 2016, no. 1, p. 9, 2016.

[3]. Prakash, M. Kumar, R. Rao Kompella, and M. Gupta, "Phishnet: prescient boycotting to choose phishing assaults," in Proceedings of 29th IEEE Conference on Computer Communications (Infocom), pp. 1-5, Citeseer, San Diego, CA, USA, March 2010.

[4]. R. M. Mohammad, L. McCluskey, and F. Thabtah, "Smart guideline based phishing sites arrangement," IET Information Security, vol. 8, no. 3, pp. 153-160, 2014.

\section{Cite this article as :}

AjithKumar Reddy K, Darshith M P, Divya Megha H S, Omshree V, Sudhakara Reddy M, "High Accuracy Phishing Detection Based on Convolutional Neural Network", International Journal of Scientific Research in Science and Technology (IJSRST), Online ISSN : 2395-602X, Print ISSN : 2395-6011, Volume 8 Issue 3, pp. 454-457, May-June 2021. Available at doi : https://doi.org/10.32628/IJSRST218393

Journal URL : https://ijsrst.com/IJSRST218393 